\title{
An Exploration of the Possibility for secondary traumatic stress amongst Transcriptionists: A Grounded Theory Approach
}

\begin{abstract}
While there is a small, growing literature that considers the psychological safety of researchers, little attention has been paid in the qualitative literature to the wellbeing of transcriptionists. Transcriptionists play an integral and essential role in qualitative research but are often overlooked in terms of the emotional impact of the work. In the article we have interviewed transcriptionists and, using grounded theory, we illustrated that that they experienced emotional distress and feelings of helplessness. We showed that while they had some internal coping mechanisms they nonetheless also expressed a need to talk about their feelings. Furthermore the general lack of safeguarding protocols made the role more challenging. At the end of the analysis the core category we identified was the risk of secondary traumatic stress. We thus made some recommendations for safeguarding transcriptionists and called for further research in this area.
\end{abstract}

Key words: Transcription, transcriber, trauma, emotional impact, qualitative, distress, secondary traumatic stress, and, coping. 


\section{An Exploration of the Possibility for secondary traumatic stress amongst Transcriptionists: A Grounded Theory Approach}

\section{Introduction}

"Primarily women, transcribers are essentially invisible persons, paid to serve as nameless, faceless technicians even though they participate in a transformative auditory experience"

(Gregory et al 1997: 294)

The research literature is filled with references to risk and in the field of health research there has been a great historical concern to protect research participants from both physical and psychological harm. In more contemporary works, these arguments have extended to express some concern with the safety of research teams. Thus, over time there has been a gradual increase in attention paid to the physical (Ensign, 2003; Sampson et al, 2008) and emotional (Dickson-Swift et al, 2009; Malacrida, 2007) safety of researchers including some clear recommendations for staying safe in the field (Bloor et al, 2007; Authors, 2013; SRA, 2010). This is particularly important in qualitative research, which requires greater engagement with participants from the research team and can be sensitive in nature. It is essential to bear in mind that in qualitative work the process and the content cannot be separated into discrete elements as they necessarily interact with one another (Bowtell et al., 2013). This literature is still growing, but arguably has paid limited attention to the wellbeing of transcriptionists.

We argue that there are four key problems for this group regarding their participation in the research process. The first issue for transcriptionists is that they tend to be the 'invisible' workers. However, transcriptionist safety is important and should not be overlooked (McCosker et al., 2001). Transcription has often been viewed as a mundane task (Lapadat \& Lindsay, 1999) and one with little status (Tilley \& Powick, 2002). For this reason it has 
typically been assumed that transcriptionists are unaffected by the process (Gregory et al., 1997). This may be the reason why very little research has been conducted on the impact of listening to qualitative data on transcriptionists. The transcriptionist, however, is not "merely an extension of the tape recorder" (McCosker et al., 2001: $11^{\text {th }}$ Page), but is a thinking, feeling human being.

The second issue for transcriptionists is the emotional impact that listening to data may cause. The process of transcription by its nature means that the transcriptionist must listen to the data repeatedly to capture the words spoken and therefore emotionally disturbing events may become embedded in their consciousness (Gregory et al., 1997). This means that transcriptionists may become emotional when listening to the data (Lalor et al, 2006) as it is challenging to stay detached when dealing with emotional and sensitive material (DicksonSwift et al., 2009). Such sensitive material is particularly likely in fields such as health, medicine, and psychology (Wilkes et al., 2014). Repeated listening to what may be traumatic stories can lead to vicarious traumatisation (Etherington, 2007) or secondary traumatic stress (author and author, 2015). Vicarious traumatisation is a fundamental transformation of the self that results from empathic engagement with trauma survivors (McCann \& Pearlman, 1990). Secondary traumatic stress is described by Devilley et al. (2009) as a 'natural consequence' of empathy between two people, one of whom has been traumatised. This is exacerbated by the repeated exposure which may cause a cumulative effect (Coles \& Mudlay, 2010). Secondary traumatic stress is the term we have applied to transcriptionists' experiences as, while similar to vicarious traumatisation, does not have the same self-transformative impact. We argue that the process of translating auditory files into written text inevitably influences the human conduit that is not immune to thoughts, feelings, memories and reactions to this auditory stimulus. For example, in a recent study with 12 transcriptionists in Australia and New Zealand, some participants found working with particular topics overwhelming and 
consequently decided not to take on any more work related to those issues (Wilkes et al., 2014).

The emotional impact has potential to lead to certain difficulties for transcriptionists, particularly as they tend to work in a more isolated manner than researchers. Thus the third issue that we present is that transcriptionists may be limited in access to appropriate support. The emotional risk is higher if they are not given an opportunity to debrief or if they have been inadequately prepared for the possible effects (Etherington, 2007). Transcriptionists are not immune to the power and sensitivity of the narratives they are exposed to but rarely are they given opportunities to talk about the emotional impact of the task (Warr, 2004). This is further complicated by the potential of breaching the confidentiality of the participants which makes it difficult for them to discuss their experiences with friends or family (McCosker et al., 2001).

Additional difficulties are caused by the lack of formal protocols for the work of transcriptionists, including briefing and debriefing, and therefore the fourth issue is the limited formal attention given to this group. While there are some guidelines for researcher safety such as the UK Social Research Association (SRA) (2010) which could translate for transcriptionists, there seems to be no legislative body or protocol in place (that we could find) specifically for transcriptionists. Typically transcriptionists are not given any formal supervision or support (Etherington, 2007) and few transcriptionists are advised about the emotional impact that may occur (McCosker et al, 2001). Evidence suggests that researchers need more specialised training, support and supervision to safeguard their emotional wellbeing (Dickson-Swift et al, 2008). We argue that this is also the case for transcriptionists, particularly for difficult psychological research topics. While we recognise that the majority of our sample were professional transcriptionists, and that it is often the case that researchers undertake their own transcription, our study focused predominantly on the experiences of non-research active transcriptionists. In this article we aim to explore this issue from the perspective of 
transcriptionists, to address the question 'what is the emotional impact of transcribing qualitative data on transcriptionists?'

\section{Methods}

A qualitative grounded theory design was adopted to examine the social psychological processes of transcriptionists working with qualitative data. While qualitative research is an approach underpinned by a range of different theoretical positions (see authors, in press) we took a broadly social constructionist approach to analysis as this promotes participants' versions of reality (Burr, 2003) and is congruent with some forms of grounded theory (Burck, 2005).

\subsection{Sample and Setting}

The sampling approach adopted was purposeful and utilised a snowball approach. This allowed us to identify transcriptionists across the UK who were currently transcribing qualitative data sets. A final sample of 9 individuals was included in the study which facilitated an in-depth exploration of their views and experiences. The transcriptionists varied in age, ranging from 25 years to retirement age and also varied in their levels of experience. The sample consisted of eight women and one man. Only one transcriptionist was also a research student.

\subsection{Data Collection and Analysis}

Semi-structured interviews were undertaken to explore transcriptionists' experiences of transcribing qualitative data. Some interviews were conducted face-to-face and others by telephone to account for geographical locations, and all were audio-recorded. Data collection 
and analysis were conducted simultaneously in line with grounded theory. This inductive approach was adopted in order to identify and explore salient issues from the perspective of the participants, which is important when little is known about a phenomenon. This required engagement in constant comparative analysis, which allowed the features to be categorised and verified to form a theory grounded in the data (see Charmaz, 2005).

No new categories were identified by the completion of case number 6, but a further 3 participants were recruited to ensure certainty. Thus theoretical saturation was achieved through the inclusion of the $9^{\text {th }}$ case, which we accept is possibly unusual after a small number of interviews and is more typically occurring at 10-12 (see Francis et al, 2010). The data-driven strategy identified 27 categories which we collapsed into a total of five themes and were included for analysis. The final core category identified was the risk of secondary traumatic stress for transcriptionists because of exposure to emotionally charged qualitative material.

\subsection{Ethics}

Ethical approval for the project was granted by the University of Leicester Ethics Committee. All transcriptionists were assured of anonymity in the transcripts and dissemination and opted into the study by providing written consent in advance of the interview.

\section{Analysis}

Strauss and Corbin (1990) proposed that it is necessary to develop categories from the raw data which require the identification of concepts contained within the data. Each interview was carefully scrutinised so that concepts could be identified and that statements containing similar words could be grouped together. This grouping thus provided the basis for core themes for 
analysis by linking the concepts and categories, and helped to reduce a large volume of data into something more meaningful (Strauss \& Corbin, 1990). The core category that finally emerged was that of 'risk of secondary traumatic stress'. This core category evolved from five themes identified from the transcriptionists' narratives. The first of these themes was the risk of emotional distress and impact on the transcriptionist. Second was the coping mechanisms used by transcriptionists. Third was feeling a sense of helplessness and/or worrying about the researcher. Fourth was their need to talk. Fifth was the lack of safeguarding protocols or guidelines.

\subsection{Theme One: The risk of emotional distress and impact on the transcriptionist}

A common issue for the transcriptionists was the potential risk to their own emotional welfare when listening to the stories told by participants on the recordings. It is very common in qualitative research for researchers to be examining the lived experiences of people's real personal lives and often this can cover sensitive or distressing issues (McIntosh \& Morse, 2009). Transcriptionists in our study described a number of emotional reactions to listening repeatedly to difficult and sensitive narratives, including topics such as palliative care, paedophilia, mental health and children's services. This theme emerged from seven categories within the data corpus and are summarised in table 1.

\section{INSERT TABLE ONE HERE}

The categories identified within this theme of emotional distress and impact demonstrated that transcriptionists were affected by the process of transcribing, and were therefore not neutral tools but had normal human reactions to hearing about other people’s suffering. Previous 
research on transcriptionists for example has indicated that during the process they experience a number of different negative emotions including depression, anger and grief (Wilkes et al., 2014). The transcriptionists in our study highlighted an elevation in their own emotional state because of the particular emotional proximity felt by hearing participants’ voices.

1. “I've done work for palliative care department work and if they're talking to people who are dying, you know, and there's a lot of, it's very upsetting, people are crying, it is, you know. I think you couldn't help by being affected by it really, even if you're, you know, a person that does that all the time and goes into these situations. I just think it would be really almost impossible to not be affected by that."

(Transcriptionist one)

2. "I've heard things that have been upsetting, especially hearing children say the way they get treated and stuff like that by the adults in their lives that's that's difficult I suppose."

(Transcriptionist nine)

The first issue that was apparent in our analysis was that the subject matter of the data being transcribed was by its nature upsetting, such as people dying or the way children are treated by the adults in their lives. Transcriptionists described how it would be 'impossible not to be affected' by the content of the participants' narratives. This illustrated that transcriptionists were impacted by the role they took in the research process. Thus, there is a risk to the emotional wellbeing of the transcriptionists, and a risk of secondary traumatic stress (Author \& Author, 2015). In our data, this was further exacerbated by listening to participants' crying on the recording. 
3. “Reading a transcript isn't as emotional as actually hearing that person say what they've got to say, how they've been treated or how much pain they're in, or, you know. And then the tears and everything that goes with it."

(Transcriptionist two)

Our analysis has shown that transcriptionists are in an unusual position in being one of the few people who hear the actual voices and expressions of emotion experienced by the participants in the recordings. Transcriptionists described empathising with how much pain participants’ experience and expressed distress at actually hearing that person and the tears that accompanied the narrative. It is clear therefore that transcriptionists had a much more intimate experience with the audio-data than those who engage later with the written transcript produced.

\subsection{Theme Two: The coping mechanisms used by transcriptionists}

Transcriptionists made reference to a range of coping strategies to help them to engage in their central task. This indicated that there were difficult emotional responses experienced that necessitated the invocation of those coping strategies, such as detachment and desensitising. Some of these strategies were used consistently throughout their transcribing careers, whereas others evolved or developed through the process of repeated exposure to distressing material. This is not too dissimilar to those used by researchers, as evidence indicates that researchers think there is a need to remain professional and detached from the research (Dickson-Swift et al., 2009). This theme of coping mechanisms emerged from five categories within the data corpus and are summarised in table 2. 


\section{INSERT TABLE TWO HERE}

Transcriptionists appeared to engage in two broad ways of coping with emotional impact. First they reported employing immediate ways of coping such as cognitive and affective strategies to transcend and rationalise their experience. Cognitive strategies included techniques such as separating the humanity from the professional task in hand by rationalising, detaching and cognitive reframing. Affective strategies included absorbing and disengaging from the data. Second transcriptionists described a process of gradual numbing the painful impact of listening to other people's distress over a period of time, leading to a desensitisation effect.

4. I kind of, I don't know, I don't know what I do, I stick it into a little compartment in my brain, and then when it crops up again, you know, I deal with it myself really.

(Transcriptionist two)

This extract is an example of how transcriptionists attempted to deal with the emotional effect of transcribing sensitive data by compartmentalising it as part of the professional process. Notably, however this process was temporary and the impact of what had been heard crops up again and thus compartmentalisation did not appear to be a permanent solution to the problem.

5. I think the ones who stick with it are the sort of people who can deal with this sort of thing and certainly become acclimatised to getting that sort of, coming across that sort of thing and I think, yes, become used to it and become prepared for it and maybe get desensitised even just due to that sort of thing.

(Transcriptionist four) 
6. but remarkably what I found as I've said that as I went along it was affecting me it now affects me less and less

(Transcriptionist eight)

Transcriptionist four (extract five) alluded to the idea of certain personality types that are better suited to managing difficulties of repeated listening to distressing narratives. For both transcriptionists four and eight there was also an underlying presupposition that the work carries an emotional toll but that this lessens over time through acclimatisation and desensitisation.

\subsection{Theme Three: A sense of helplessness and/or worrying about the researcher}

Transcriptionists’ accounts included descriptions about their lack of control and feeling of helplessness to help either the participants or the researchers in worrying situations. This seemed to indicate that although transcriptionists were perceived to be on the periphery of the research process, in reality they were affected by the emotional content of the data. The lack of attention paid to the role of the transcriptionists means that they remain marginalised not only from the products of their work, but also there is little recognition of the emotional labour associated with their productivity (Gregory et al., 1997). Not only does 'emotional labour' (dealing with the face-to-face/voice-to-voice contact) have emotional consequences for transcriptionists, but so does the 'emotional work' (dealing with the emotions of others) (Hoschild, 1983). In our data corpus, transcriptionists expressed empathy and concern for researchers' physical and emotional wellbeing and reported a need for researchers to be 
supported in the research process. Their distance from the data collection process, however, often left transcriptionists feeling disempowered and lacking control. This theme emerged from five categories within the data corpus and are summarised in table 3.

\section{INSERT TABLE THREE HERE}

As aforementioned, our data revealed that transcriptionists not only felt empathy and concern for participants but also for the emotional and physical wellbeing of the researcher involved in the original data collection process. The position of the transcriptionist as one who is separated from the original research situation led to a sense of frustration and helplessness, without the context and the full information about the research project.

7. but want you kind of want that final answer I think you, you form an alliance with the people your recording even though you don't know them. Yeh, umm and I think you want that answer as much for them as you do for yourself

(Transcriptionist seven)

Notably, transcriptionist seven spoke about forming an alliance with the researcher and participants on the recordings. It appeared that this personal connection precipitated a human involvement and empathy with the participants that transcended the mere mechanics of putting the words on paper. Consequently the transcriptionist was left with a feeling of incompleteness and of not knowing what the outcome of the situation was.

8. But there is a feeling of, that you can't do anything about it. Nobody nobody can really, can they? 
A recurrent issue for transcriptionists was a feeling of helplessness which suggested a sense of personal engagement with the emotional lives of participants. When listening to concerning narratives transcriptionists expressed feeling trapped between a natural emotional response of worry and the real constraints of participant anonymity and physical distance. This reflects how researchers sometimes feel about the research process as they might experience guilt for judging participants or frustration that they were unable to help or intervene (Malacrida, 2007).

\subsection{Theme Four: Transcriptionists' need to talk}

An important issue raised by transcriptionists was their felt need for practical and emotional support, and consideration of the personal impact of the job. A significant restriction on where transcriptionists can access this support, however, is the constraint of the confidentiality of the qualitative material. Previous research has shown that transcriptionists expressed a need for debriefing but none of them had experienced any form of official debriefing (Wilkes et al., 2014).Transcriptionists in our data also expressed a need to talk about their experiences and some reflected on informal mechanisms that were utilised, while others expressed an absence of appropriate avenues for support. This theme emerged from six categories within the data corpus and are summarised in table 4.

\section{INSERT TABLE FOUR HERE}

Transcriptionists are bound by the same rules of confidentiality as the research team, but the data illustrated that transcriptionists did not always think that they had the level of emotional support that they needed to handle some of the more distressing aspects of the work. In these 
cases transcriptionists expressed a conflict between the constraints of confidentiality and the need to talk to someone about their experiences.

9. I think a lot of people do it on a freelance basis and that's quite a worry because they would be very isolated and for confidentiality reasons, they wouldn't be able to talk to a partner or a third party, in fact anyone.

(Transcriptionist four)

10. I speak to my partner and that's it, because he's here when sometimes when I'm doing the work, or at night time I'm upstairs and I'm doing it and I'll say, oh it's really quite tough, this person's really upset but obviously you don't break confidentiality because I can't.

(Transcriptionist two)

In both of the examples above the transcriptionists expressed a dilemma about needing to talk to someone but not having formal mechanisms in place, and therefore coming to their own decisions regarding the appropriateness of sharing with family members. For example, transcriptionist four reported that it could be isolating because of the position taken that transcriptionist cannot talk to a partner or a third party. Transcriptionist two, however, expressed that their partner was the person turned to at night time, but that this was constrained by confidentiality. This raises the issue that often transcriptionists were not working traditional 9-5 hours within an office environment, and therefore their needs need to be considered more flexibly.

11. I've been in situations where I have had direct contact with the researcher, I think it's, you know, I think it's really beneficial for both parties, not just for me. I think it is for 
them as well. 'Cos it just builds up a relationship that, you know, includes you as part of the process that's happening, rather than some detached peripheral part of it. I think, you know, in the instance where I just deal with the admin staff, I'm just kind of this peripheral, outsourced person

(Transcriptionist one)

Transcriptionist one expressed a view which was not uncommon in the data regarding feeling peripheral to the research process. Often transcriptionists are self-employed individuals who take on transcription work through agencies or as independent persons and are therefore are not working within a research environment. Transcriptionist one described how helpful and beneficial a direct relationship with the researcher was in helping her to be less detached and peripheral. It appears that this inclusion in the wider research process was perceived to have benefits to both transcriptionists and for researchers.

\subsection{Theme Five: Need for protocols or guidelines}

Transcriptionists expressed the view that there was a need for clearer protocols regarding the transcription process, the need for informed briefing procedures regarding the content of the data and more formal support and debriefing mechanisms. In particular transcriptionists expressed their perspective that an important part of the process should be that they are briefed about any potentially distressing content to the recordings that they are transcribing. This would facilitate an opportunity to make an informed decision regarding taking on the role, but would also better prepare them for any potential emotional impact. This theme emerged from four categories within the data corpus and are summarised in table 5. 
From personal experience, transcriptionists were able to advocate that the specific administrative task of typing audio material is much more than simply a clerical role. The transcriptionists in our sample clearly expressed their views that transcribing qualitative research data has specific aspects that need particular consideration and attention.

12. it's definitely important to know what you're getting into, not to think of it as a typing job, think of it, um, as something that will have repercussions that you will wake up in the night and think about on a level that you just wouldn't expect from that sort of, that level of clerical work really.

(Transcriptionist four)

Unlike traditional clerical work which might be of fairly neutral content, research transcription in contrast is described by transcriptionists as something that will have repercussions and could lead them to wake up in the night. Thus, experienced transcriptionists in our study recommended that people new to this type of transcription work should be made aware of the potential emotional impact because of the sensitive data.

13. I think one possible area which may be useful in that situation is for the researcher to actually indicate to the transcriber, if anything comes up in the course of the transcription which you feel uneasy about then, you know speak to me.

(Transcriptionist five)

14. I'd definitely want to put something in place, some sort of counselling or, you know, kind of occupational health type thing, if I ran a company that was big enough to warrant it 
In addition to preparation, transcriptionists also made reference to the need for debriefing by either having the researcher available to discuss the effect that the content has had on them or an independent counsellor. Because of the confidentiality of the work and the ethical requirements for anonymity for participants, transcriptionists are often constrained in terms of who they can talk to.

\section{The Core Category: The Potential Risk of Secondary Traumatic Stress}

The core category that emerged from the five themes that were discovered through analysis was that there appeared to be a potential risk of secondary traumatic stress among transcriptionists who were involved in transcribing qualitative data, particularly that of a sensitive or emotive content. The key concerns that transcriptionists raised fell into a number of overlapping and interrelated themes predominantly focused around the emotional impact of transcribing and the need for support. Transcriptionists spoke about the distressing nature of the content of data, and feelings of helplessness and concern about the participants and the researchers. They discussed a need to talk about their experiences, but that the constraints of confidentiality limited the scope of who they could turn to. In the absence of formal protocols regarding the briefing and debriefing of transcriptionists, our participants conveyed a number of unofficial coping strategies that were more or less effective. The theory that we have proposes, therefore from this empirical investigation, is that 'transcriptionists are at risk of secondary traumatic stress and require formal support either from a governing body for transcriptionists and/or by being recognised more explicitly as part of the research team'. 


\section{Discussion}

Our interest in the impact of listening to recordings of sensitive, emotional, or distressing material on transcriptionists was precipitated by personal experiences of working with transcriptionists who were transcribing qualitative data. These encounters triggered an interest to explore further whether this was a frequent and common experience; out of which was borne our review of the existing literature which proved to be limited in scope. We were, therefore, prompted to investigate the experiences of transcriptionists on a more formal basis by developing a research project.

Through the sampling method and data collection process it became apparent that transcriptionists represented a diverse body with different working procedures and practices. Despite this diversity, however, they all expressed views regarding the 'invisibility' of their role in the process, and saw themselves as relatively peripheral to the research team. Our findings therefore confirmed the view expressed in the literature that transcriptionists are on the periphery of the research process (Gregory et al, 1997).

A second problem identified through our analysis was that transcriptionists felt an empathic connection with the participants whose voices they were listening to as they transcribed the data. This was further worsened by also listening to participant's crying and their emotional displays. This was often an unexpected reaction that transcriptionists felt they were ill-prepared for. This is not dissimilar to researchers who sometimes naively think that they might be able to detach themselves from the situation but then find that they cannot (Hubbard et al, 2001). The combination of the two vulnerability factors of isolation and emotional impact, led to a third issue of the risk of secondary traumatic stress. This appeared to be especially problematic where transcriptionists were listening repeatedly to emotional data. 
This negative impact on the wellbeing of transcriptionists has previously tended to be mostly unrecognised and unacknowledged by the qualitative community and thus, this research has highlighted an important and timely issue. It appears from our data that the transcriptionists had limited or inadequate formal support in managing the impact of exposure to distressing material from research teams or from a professional governing body. This is the essence of the fourth problem, that there are no official safeguarding protocols for this working group.

We therefore propose that it would be beneficial for research teams, particularly in qualitative research in psychology where emotive and/or sensitive topics are being explored, to take a more proactive role in considering the emotional needs of transcriptionists that they employ. We recognise there is a diversity of circumstances in which transcriptionists are commissioned to undertake qualitative transcribing activities. Additionally the limited personal contact between transcriptionists and researchers can made it difficult for researchers to be involved in transcriptionist's welfare (Etherington, 2007). Nonetheless, there is potentially still scope for researchers to actively prepare and support transcriptionists in their role. In some circumstances it might be appropriate and/or possible for the transcriptionist to be considered as a more integral part of the research team. Where this is not practical or appropriate, we suggest that researchers still have an obligation to offer both briefing and debriefing opportunities for their transcriptionists. While not all researchers consider themselves equipped to offer emotional support to transcriptionists (Lee-Treweek \& Linkogle, 2000), because of the boundaries of confidentiality for research participants, researchers are in effect the only individuals in a position to discuss the details of the content of the data and therefore to fulfil this role. We acknowledge that in some teams this already forms part of the research procedure and would support this as good practice. We would therefore encourage researchers to more publicly share their experiences. We also suggest that questions about safety for ethics committees should be further reaching than they currently manage, and should include the research team and transcriptionist, as well as the participants. This was an 
important point made in the Inquiry conducted by Bloor et al (2007) in relation to researcher safety.

We acknowledge that there are some limitations to the present study which could reduce its transferability to other qualitative settings. Due to the exploratory nature of the design, by necessity the sample size was ostensibly small. Nonetheless, saturation was reached and a number of important issues have been identified which will provide an important benchmark for additional research. We recognise that our sample were all from various counties within the UK, and thus have offered a particularly British perspective on this new problem. However we anticipate that this is an issue which is likely to be internationally comparable. We would, therefore, encourage qualitative scholars from other countries to engage in equivalent research so that synthesis of the issues might be managed. Similarly, we anticipate that there may be a degree of transferability across professions, as there is already an evidence-base that indicates that secondary traumatic stress and vicarious traumatisation are experienced not only by psychological therapists (McCann \& Pearlman, 1990), but also (amongst others) by asylum lawyers (Piwowarczyk et al., 2009), members of the clergy (Day et al., 2006), social service workers (Pryce et al., 2007), and interpreters (Harvey, 2001).

In conclusion, our investigation has revealed that transcriptionists are considerably emotionally affected by the task of repeated listening and transcribing of data. This was more surprising than we initially anticipated when taking on this project. Additionally, while maintaining the anonymity and confidentiality of the research participants is necessarily paramount, it appears that the requirement for confidentiality creates some problems for the transcriptionists. First is that this limits who they can talk to about their emotional experiences and second is that transcriptionists reported feeling helpless regarding the distressing material that they could neither influence nor have closure about. The theoretical conclusion that our research has indicated is that transcriptionists are potentially susceptible to secondary traumatic stress through their professional activities. Therefore transcriptionists could benefit from being 
more integrated within the research team and their personal and emotional needs should be recognised as well as their technical skills. 


\section{References}

Bloor, M Fincham, B \& Sampson, H (2007) QUALITI (NCRM) Commissioned inquiry into the risk and well-being of researchers in qualitative research. ESRC, Cardiff

Bowtell, E Sawyer, S Aroni, R Green, J \& Duncan, R (2013) “Should I send a condolence card?” Promoting emotional safety in qualitative health research through reflexivity and ethical mindfulness. Qualitative Inquiry, 19(9), 652-663

Burck, C (2005) Comparing qualitative research methodologies for systemic research: The use of grounded theory, discourse analysis and narrative analysis. Journal of Family Therapy 27: 237262.

Burr, V (2003) Social constructionism (second edition). Routledge, London.

Charmaz, K (2005) Grounded theory in the $21^{\text {st }}$ Century: applications for advancing social justice studies. In: N Denzin \& Y Lincoln (Eds.) The SAGE Handbook of Qualitative Research (Third edition). Sage, London, pp. 507535

Coles, J \& Mudlay, N (2010) Staying safe: strategies for qualitative child abuse researchers. Child Abuse Review 19, 56-69.

Day, J Vermilyea, E Wilkerson, J \& Giller, E (2006) Risking connection in faith communities: A training curriculum for faith leaders supporting trauma survivors. Sidran Institute Press, Baltimore, MD.

Dickson-Swift, V James, E Kippen, S \& Liamputtong, P (2008) Risk to researchers in qualitative research on sensitive topics: issues and strategies. Qualitative Health Research 18 (1): 133144.

Dickson-Swift, V James, E Kippen, S \& Liamputtong, P (2009) Researching sensitive topics: qualitative research as emotion work. Qualitative Research 9 (1): 61-79. 
Ensign, J (2003) Ethical issues in qualitative health research with homeless youths. Journal of Advanced Nursing 43 (1): 43-50.

Etherington, K (2007) Working with traumatic stories: from transcriber to witness. International Journal of Social Research Methodology 10 (2): 85-97.

Francis, J Johnston, M Robertson, C Glidewell, L Entwistle, V Eccles, M \& Grimshaw, J (2010) What is adequate sample size? Operationalising data saturation for theory-based interview studies. Psychology and Health 25 (10): 1229-1245

Gregory, D Russell, C \& Phillips, L (1997) Beyond textual perfection: Transcribers as vulnerable persons. Qualitative Health Research 7 (2): 294-300.

Harvey, M (2001) The hazards of empathy: Vicarious trauma of interpreters for the deaf. Journal of Interpretation, 11, 85-98.

Hochschild, A (1983) The managed heart: commercialization of human feeling, with a new afterword. University of California Press, Berkley, CA.

Lalor, J Begley, C \& Devane, D (2006) Exploring painful experiences: impact of emotional narratives on members of a qualitative research team. Journal of Advanced Nursing 56 (6): 607-616.

Lapadat, J \& Lindsay, A (1999) Transcription in research and practice: from standardization of technique to interpretive positioning. Qualitative Inquiry 5 (1): 64-86.

Malacrida, C (2007) Reflexive journaling on emotional research topics: Ethical issues for team researchers. Qualitative Health Research 17 (10): 1329-1339.

McCann, I L \& Pearlman, L (1990a). Vicarious traumatization: A framework the psychological effects of working with victims. Journal of Traumatic Stress, 3(1), 131-149.

McCosker, H Barnard, A \& Gerber, R (2001) Undertaking sensitive research: Issues and strategies for meeting the safety needs of all participants. Forum: Qualitative Social Research 2 (1): Online.

McIntosh, M \& Morse, J M (2009) Institutional review boards and the ethics of emotion. In: NK Denzin \& M D Gardina (Eds.), Qualitative Inquiry and Social Justice. Left Coast Press, Walnut Creek, CA, pp: 81-107 
Authors (2013)

Authors (in press)

Piwowarczyk, L Ignatius, S Crosby, S Grodin, M Heeren, T \& Sharma, A (2009) Secondary trauma in asylum lawyers. Bender’s Immigration Bulletin, 14(5), 263-269.

Pryce, J Shackelford, K \& Price, D (2007) Secondary traumatic stress and the child welfare professional. Lyceum Books, Inc, Chicago.

Sampson, H Bloor, M \& Fincham, B (2008) A price worth paying? Considering the 'cost' of reflexive research methods and the influence of feminist ways of 'doing'. Sociology 42 (5): 919-933.

Social Research Association. 'A Code of Practice for the Safety of Social Researchers'. As retrieved from www.the-sra.org.uk on $18^{\text {th }}$ October 2010.

Strauss, A \& Corbin, J (1990) Basics of Qualitative Research: Grounded Theory Procedures and Techniques. Newbury Park, CA: Sage.

Tilley, S \& Powick, K (2002) Distanced data: Transcribing other people’s research tapes. Canadian Journal of Education 27 (2\&3): 291-310.

Warr, D (2004) Stories in the flesh and voices in the head: reflections on the context and impact of research with disadvantaged populations. Qualitative Health Research 14 (4): 578-587.

Wilkes, L Cummings, J \& Haigh, C (2014) Transcriptionist saturation: Knowing too much about sensitive health and social data. Journal of Advanced Nursing, DOI: 10.111/jan.12510

No funding was received for this project. 